\title{
Ecomoulding of Composite Wind Turbine Blades Using Green Manufacturing RTM Process
}

\author{
Brahim Attaf \\ Team Europe, European Commission, 39 Boulevard Charles Moretti A6, 13014 Marseille, France \\ Correspondence should be addressed to Brahim Attaf, b.attaf@wanadoo.fr \\ Received 3 May 2012; Accepted 17 July 2012 \\ Academic Editors: W. Han and A. Martinez-Villafane \\ Copyright () 2012 Brahim Attaf. This is an open access article distributed under the Creative Commons Attribution License, which \\ permits unrestricted use, distribution, and reproduction in any medium, provided the original work is properly cited. \\ According to ecodesign considerations and green manufacturing requirements, the choice of moulding process for the production \\ of composite wind turbine blades must provide the existence of a common area of intersection engendered by a simultaneous \\ interaction between quality, health, and environment aspects (i.e. $Q, H$, and $E$ for abbreviations, resp.). This common area can \\ be maximized via ecoalternatives in order to minimize negative adverse environmental and/or human health impacts. With this \\ objective in mind, the closed-mould manufacturing RTM (resin transfer moulding) process will be the subject matter of this \\ investigation. The reason for this choice is that RTM process participates in the reduction of VOC (volatile organic compound) \\ emissions such as styrene vapours and presents an industrial solution to wind turbine blades production coupled with high quality \\ finishing, good mechanical properties, lower cost, and a total absence of bonding operation of half shells. In addition to these \\ advantages, sustainable development issues and ecodesign requirements are still, however, the main objectives to be fulfilled in \\ this analysis with an acceptable degree of tolerance to the new regulations and ecostandards leading the way for green design of \\ composite wind turbine blades.
}

\section{Introduction}

In the modern manufacturing industry of wind turbine blades [1], fibre-reinforced composite materials are chosen among other available engineering materials because of their significant and attractive advantages in terms of stiffnessand strength-to-weight ratio, thermal and chemical resistance properties, coupled with material cost effectiveness [2]. In addition to the economic advantage and technicalquality efficiency, further ecological aspects are still required in order to green up the moulding processes and this after an appropriate selection of matrix resin and fibre materials that are answering questions related to environmental and safety issues. These ecological aspects are based on balanced key criteria characterized mainly by a comprehensive consideration of environmental preservation and health protection besides quality assurance [3-5]; all of which must provide healthier, safe, clean, and sustainable process.
Within this environmentally mindful context, the closed mould process provides an alternative solution to these requirements and satisfies the condition of green design method (i.e., ecodesign).

Further to that, the advantages achieved by the green moulding processes (e.g., closed mould process) with regard to other traditional moulding processes (e.g., open mould process) are performed at three levels of environmental consciousness, which of these focused on (i) the improvement of competitiveness and productivity via innovative engineering approaches, (ii) the minimization of energy consumption, and (iii) the reduction of emission levels via alternative solutions.

This new mode of composite manufacturing can greatly enhance the development of ecoefficient and reliable moulding processes and can therefore help boost potential development level for industrial wind energy field at short, medium, and long term. Among these closed-moulding processes, 
the most familiar one is the resin transfer moulding (RTM) process [6]. The present study will focus on techniques and formulation of resin flow in an anisotropic fibrous medium. Although some background analysis techniques of the state-of-the-art related to this subject can be found in the literature, [7] has addressed some very important research studies on the flow and rheology in polymer composites manufacturing.

By adopting the strategy in the context to go green can provide a sustainable moulding process which may reinforce the technological programme of Wind Energy Roadmap [1] and may help composite wind turbine blades manufacturers to achieve the following key ecodesign aims: (i) reduction of VOC emissions, (ii) use of nontoxic chemicals, (iii) use of no carcinogenic substances, (iv) generation of gelcoats with low emission of odours, and so forth.

With this objective as a key target, a further aim of this study is to send a strong message to nongreen composite companies to encourage them to join the green movement by setting up new strategies to reform the old manufacturing practices.

In one hand, this ecoaction will provide sustainable methods to develop new products with very cost-effective way for wind energy activities; while on the other hand, it will play a key role in stimulating innovation, creativity, and competitiveness in the worldwide industry of wind turbine blades.

\section{Methodology of Ecomoulding}

The current scientific research investigation is directed towards a methodology of analysis to provide an ecoefficient moulding process that minimizes environmental impact and ensures health protection whilst maintaining quality assurance criterion. This ecocompatible solution can help designers and analysts to assess and improve environmental and health aspects.

2.1. Concept of Ecomoulding. The diagram shown in Figure 1 represents the ecomoulding model as an interaction between quality, health, and environment aspects. As it can be seen from Figure 1, this interaction yields a certain number of subsets. However, only subset $\ddot{F}$ is fulfilling the required condition of ecomoulding. The three dots $(\therefore)$ above the character " $F$ " are only a brief description of the diagram illustrated in Figure 1, showing interaction between health, quality, and environment aspects (i.e., $\therefore$ ). In other terms, the three dots represent the three pillars that characterize the basic elements of the sustainable development concept $[8]$.

2.2. Application of Probability Approach. To evaluate the number of chances providing the realization of the event $\therefore \dot{F}$ (subset of the event), it is necessary to use the notion

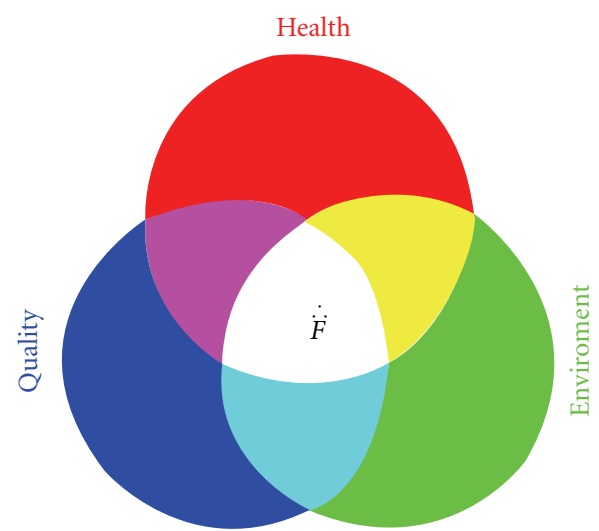

Figure 1: Model of ecomoulding process.

of probability. Therefore, the event $\ddot{F}$ and the associated probability, denoted $P(\dot{F})$, can be written respectively as [9]

$$
\begin{gathered}
\dot{F}=Q \cap H \cap E, \\
P(\dot{F})=P(Q \cap H \cap E) .
\end{gathered}
$$

According to the dependency of sets $Q, H$, and $E$ and the rules of multiplication in the probability theory, (2) can be written as follows:

$$
P(\dot{\vec{F}})=P(Q) \times P_{Q}(H) \times P_{Q \cap H}(E) .
$$

As the moulding process depends on the probability value expressed by (3), it is convenient to assign to each of $Q, H$, and $E$ aspects a specific coefficient representing the probability of approval. In the searched case, it may therefore be considered that

(i) $\alpha=P(Q)$ is an eco-coefficient representing the probability of approval in terms of quality assurance;

(ii) $\beta=P_{Q}(H)$ is an eco-coefficient representing the probability of approval with regard to health protection known that quality is achieved;

(iii) $\gamma=P_{Q \cap H}(E)$ is an eco-coefficient representing the probability of approval with regard to environmental preservation, known that health and quality are achieved.

The condition to green up the moulding process (subset $\dot{F}$ as shown in Figure 1) is performed by considering the mathematical product of the above-mentioned ecocoefficients. For notation simplicity, the quantity obtained by the rule of multiplication may be represented by a single variable called "ecofactor" and denoted by the Greek letter $\lambda$.

According to this approach, (3) can therefore be written as

$$
\lambda=\alpha \times \beta \times \gamma
$$




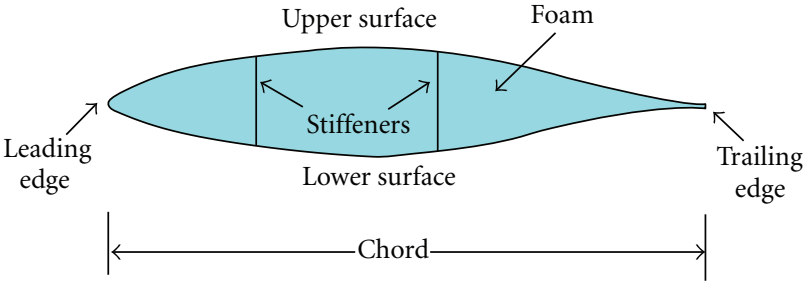

FIgURE 2: Airfoil-shaped cross section.

This ecofactor is considered to be as a key performance indicator (KPI). It is made for the purpose of discussion and analysis and will be used to provide better assessment of Q-H-E performance in relation to the moulding process. For instance, if the ecofactor $\lambda$ approaches unity $(100 \%)$, the process used for moulding is fully satisfying the green design requirements and ensuring its sustainability. However, if the ecofactor $\lambda$ is not close to the target value required by sustainability standards, it is recommended to search for possible new alternatives that provide new eco-coefficients and then new derived ecofactor. Table 1 recapitulates the assessment operation for different intervals and shows a rating satisfaction measure in the form of colour gauges.

\section{Blade Structure, Material, and Mechanical Characterization}

3.1. Sandwich Structure. The design and selection procedure of the airfoil section are discussed in [10] by considering the basic aerodynamic theory. In the current research study, the airfoil section of the typical blade is a sandwich structure separated by a thick lighter element called core (polystyrene). The upper and lower surfaces (skin of the blade) are manufactured from glass-fibre reinforced laminates. To further increase the longitudinal blade stiffness, two composite longitudinal stiffeners were incorporated into the internal structure of the sandwich, as shown in Figure 2. The stiffeners are located at 25\% C and 55\% C with regard to the blade leading edge, where the letter $\mathrm{C}$ characterizes the chord of the considered airfoil (see Figure 2). Stiffeners are made of the same material used for upper and lower laminated surfaces.

3.2. Mechanical Characteristics. Blade structure requires high strength and high stiffness in the longitudinal directions and this dictates that the majority of fibres should be uniaxially (or nearly uniaxially) aligned but that some hoop strength should also be provided. The moduli of elasticity for layers parallel and perpendicular to the fibres are denoted by $E_{1}$ and $E_{2}$, respectively. The blade skin is manufactured by several stacking sequences of unidirectional E-glass fibre (UD $900 \mathrm{~g} / \mathrm{mm}^{2}$ ), orientated principally in the $0^{\circ}$ direction (along the blade length) with some layers orientated at $\pm 45^{\circ}$.

The properties of the composites were based on $60 \%$ fibre volume fraction. The material properties are listed in Table 2.

3.3. Geometry and Dimensions. Figure 3 illustrates the principal lateral dimensions of a wind turbine blade. The
TABLe 1: Probability colour gauges for different eco-factor values of $\lambda$.

\begin{tabular}{lcc}
\hline Interval & Assessment & Gauge \\
\hline$\lambda_{5} \leq \lambda \leq 1$ & Excellent & $-\lambda_{5}$ \\
$\lambda_{4} \leq \lambda<\lambda_{5}$ & Very good & $-\lambda_{4}$ \\
$\lambda_{3} \leq \lambda<\lambda_{4}$ & Good & $-\lambda_{3}$ \\
$\lambda_{2} \leq \lambda<\lambda_{3}$ & Fair & $-\lambda_{2}$ \\
$\lambda_{1} \leq \lambda<\lambda_{2}$ & Poor & $-\lambda_{1}$ \\
$0 \leq \lambda<\lambda_{1}$ & & -0 \\
\hline
\end{tabular}

TABLE 2: Composite material properties.

\begin{tabular}{lccccc}
\hline Material & \multicolumn{5}{c}{ Material properties } \\
& $E_{1}(\mathrm{MPa})$ & $E_{2}(\mathrm{MPa})$ & $G_{12}(\mathrm{MPa})$ & $v_{12}$ & $\begin{array}{c}\text { Density } \\
\left(\mathrm{kg} \cdot \mathrm{m}^{-3}\right)\end{array}$ \\
\hline UD99/Epoxy & 25350 & 6265 & 2235 & 0.35 & 4.0 \\
\hline
\end{tabular}

blade structure was divided into four zones with different thicknesses; thus four material zones were considered. Each material is affected to the corresponding zone. On the other hand and according to stress distributions along the blade length, a decrease in thickness from blade root to free end was considered for each zone. In addition, a fifth material zone was considered for the two internal stiffeners; each one has a thickness of $3 \mathrm{~mm}$. Figure 3 illustrates how the different zones of materials and their positions relative to the blade structure were sectioned. These are defined as follows:

(i) Zone A corresponds to the upper or lower surface area with a thickness of $12 \mathrm{~mm}$.

(ii) Zone B corresponds to the upper or lower surface area with a thickness of $9 \mathrm{~mm}$.

(iii) Zone $\mathrm{C}$ corresponds to the upper or lower surface area with a thickness of $6 \mathrm{~mm}$.

(iv) Zone D corresponds to the upper or lower surface area with a thickness of $3 \mathrm{~mm}$.

\section{RTM Moulding Process}

This technique of moulding involves injecting the resin in liquid state into a closed mould cavity, in which the dry fibre reinforcements (glass-fibres preform) that were previously placed rely primarily on pressure difference that occurs inside the closed cavity, allowing the resin to flow and therefore impregnate the preformed dry reinforcements $[11,12]$. Figure 4 illustrates the sequence of the RTM process, which is summarized by the following stages:

Stage 1. selection of fibre reinforcements (and matrix resin) recommended by the design office. 

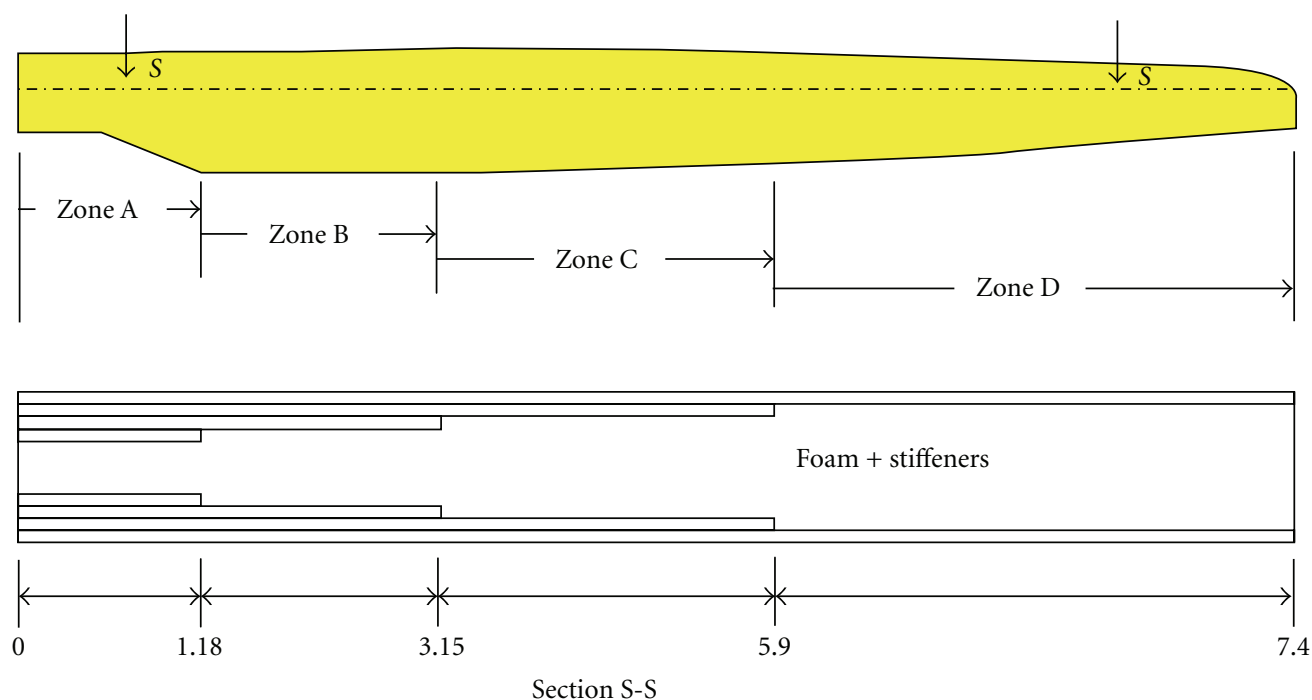

FIgURE 3: Different thicknesses assigned to different zones.

Stage 2. preparation of fibrous preform (orientation of fibres and stacking sequence).

Stage 3. placement of fibrous preform, closing the mould and venting operation.

Stage 4. injection of the resin, progressive flow of resin and impregnation of the fibre bed until filling.

Stage 5. polymerisation process, drying and hardening of the resin (curing).

Stage 6. opening the mould and demoulding the composite blade part.

Stage 4 appears to be an important step in the production process of composite blades. This step of injection and flow of a resin through the fibrous medium is based on the use of Darcy's law [13] which is mainly governed by the permeability value of the resin $K$, a physical characteristic representing the capacity of resin transfer through the fibrous material selected [14]. Further to that, this permeability depends on several factors such as the nature of the reinforcement, the direction and arrangement of fibres, the stacking sequence of plies, the temperature of the resin, the position of injection-vent ports, and so forth. Therefore, the simulation of flow behaviour in an anisotropic fibrous medium [15] must be studied carefully and the permeability values have to be correctly defined, because a minor error in the calculation of these values can lead to considerable variations that cannot be accepted in practice.

\section{Formulation of the Permeability (Darcy's Law)}

5.1. Principle of Measuring Permeability (One-Dimensional Flow 1D). In 1856, Darcy has shown that for a Newtonian incompressible fluid in laminar flow, the speed at which the fluid behaves in a homogeneous isotropic medium is proportional to the pressure gradient and inversely proportional to its dynamic viscosity [13]:

$$
v=\frac{Q}{S}=\frac{K}{\mu} \times \frac{\Delta P}{\Delta L}
$$

where, $v$ is the fluid velocity $\left(\mathrm{m} \cdot \mathrm{s}^{-1}\right) ; \mathrm{Q}$ is the discharge rate $\left(\mathrm{m}^{3} \cdot \mathrm{s}^{-1}\right) ; S$ is the cross-sectional area to flow $\left(\mathrm{m}^{2}\right) ; K$ is the permeability of the medium $\left(\mathrm{m}^{2}\right) ; \mu$ is the fluid viscosity $(\mathrm{Pa} \cdot \mathrm{s}) ; \Delta P$ is the pressure difference $(\mathrm{Pa}) ; \Delta L$ is the length of the porous medium $(\mathrm{m})$.

Using the pressure gradient notation (i.e., $\nabla P=\Delta P / \Delta L$ ) leads to

$$
v=\frac{K}{\mu} \times \nabla P .
$$

5.2. Longitudinal and Transverse Permeabilities (ThreeDimensional Flow 3D). The relation expressed by (5b) can be generalized in a three-dimensional system, as illustrated in Figure 5.

Consequently, in the three-dimensional resin flow (3D), the generalized Darcy's law can be written in the following compact form $[16,17]$ :

$$
\bar{v}=-\frac{1}{\mu}[K] \nabla P
$$

or in the following developed form:

$$
\left\{\begin{array}{c}
v_{x} \\
v_{y} \\
v_{z}
\end{array}\right\}=-\frac{1}{\mu}\left[\begin{array}{lll}
K_{x x} & K_{x y} & K_{x z} \\
K_{y x} & K_{y y} & K_{y z} \\
K_{z x} & K_{z y} & K_{z z}
\end{array}\right]\left\{\begin{array}{l}
\frac{\partial P}{\partial x} \\
\frac{\partial P}{\partial y} \\
\frac{\partial P}{\partial z}
\end{array}\right\}
$$


(1) Dry reinforcement

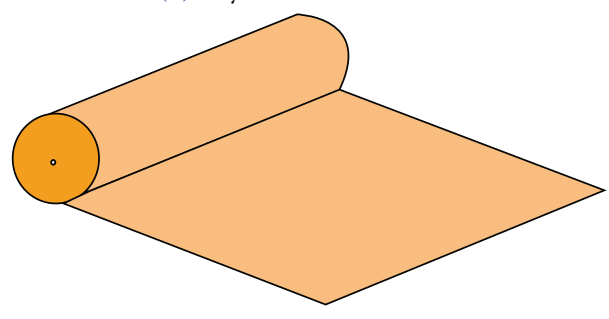

(3) Closing mould

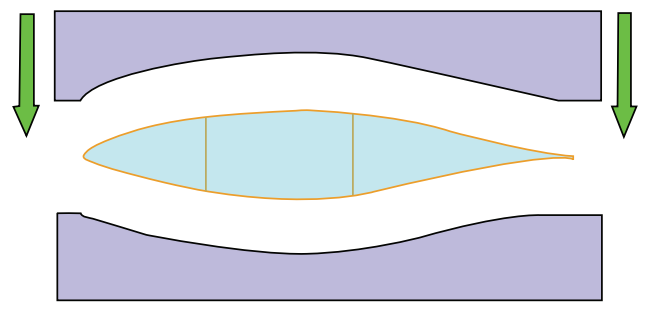

(5) Cure

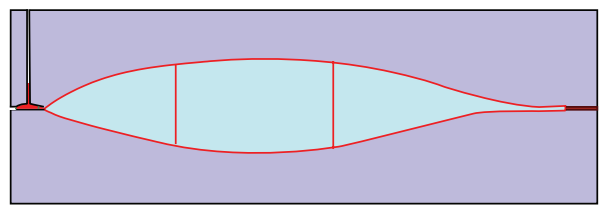

(2) Preform

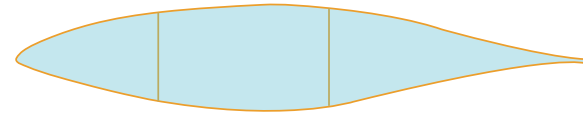

(4) Injection

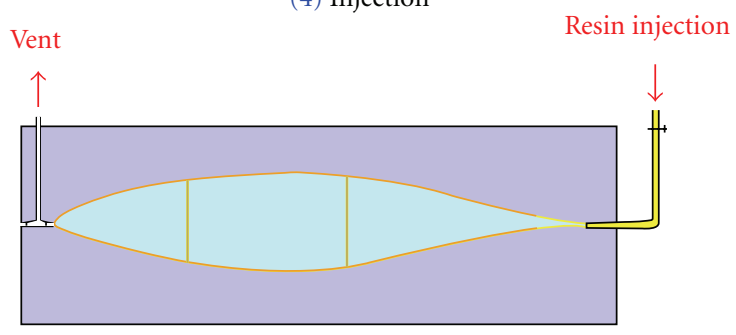

(6) Demoulding

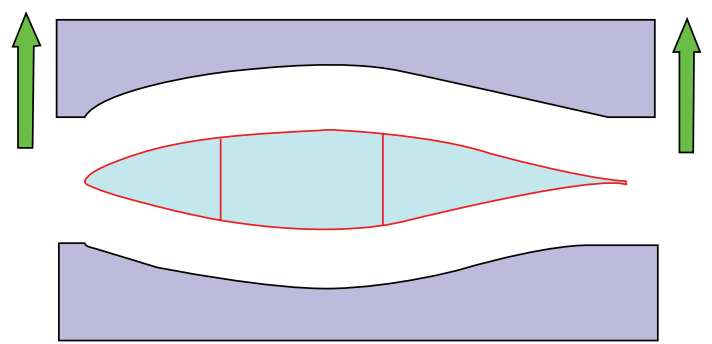

FiguRE 4: Different stages for the production of a composite blade part by the RTM process.

where $\bar{v}$ is the velocity vector $\left(\mathrm{m} \cdot \mathrm{s}^{-1}\right) ;[K]$ is the permeability tensor $\left(\mathrm{m}^{2}\right) ; \nabla P$ is the pressure gradient $\left(\mathrm{Pa} \cdot \mathrm{m}^{-1}\right)$.

In most cases, composite blades have thin laminated thicknesses in comparison to their other dimensions (length and width). Therefore, the transverse permeability through the preform thickness can be neglected. Based on this assumption, (6b) can be written in the two-dimensional flow system (2D) as

$$
\left\{\begin{array}{l}
v_{x} \\
v_{y}
\end{array}\right\}=-\frac{1}{\mu}\left[\begin{array}{ll}
K_{x x} & K_{x y} \\
K_{y x} & K_{y y}
\end{array}\right]\left\{\begin{array}{l}
\frac{\partial P}{\partial x} \\
\frac{\partial P}{\partial y}
\end{array}\right\} .
$$

As the permeability tensor $K$ depends on the angle of fibre orientation $\theta$ (as shown in Figure 6), it can therefore be written as [17].

(i) In the $(1,2)$ principal coordinate system:

$$
\left[\begin{array}{ll}
K_{x x} & K_{x y} \\
K_{y x} & K_{y y}
\end{array}\right]=\left[\begin{array}{cc}
K_{11} & 0 \\
0 & K_{22}
\end{array}\right]
$$

(ii) In the $(x, y)$ general coordinate system:

$$
\left[\begin{array}{ll}
K_{x x} & K_{x y} \\
K_{y x} & K_{y y}
\end{array}\right]=\left[\begin{array}{cc}
K_{11} C^{2}+K_{22} S^{2} & \left(-K_{11}+K_{22}\right) C S \\
\left(-K_{11}+K_{22}\right) C S & K_{11} S^{2}+K_{22} C^{2}
\end{array}\right] \text {, }
$$

where, $C=\cos (\theta)$ and $S=\sin (\theta)$.

Also, the permeability in $(1,2)$ system can be evaluated, from Carman-Kozeny equation [18], as follows:

$$
K_{i j}=\frac{1}{k_{i j}} \frac{R_{f}^{2}}{4} \frac{\left(1-V_{f}\right)^{3}}{V_{f}^{2}} \quad(i, j=1,2),
$$

where $k_{i j}$ is Kozeny constant; $R_{f}$ is the fibre radius; $V_{f}$ is the fibre volume fraction.

On the other hand, the average permeability components $\bar{K}_{i j}$ for a preform composed of $n$ plies each of thickness $h^{I}$ can be calculated according to the following rule of superposition [17]:

$$
\bar{K}_{i j}=\frac{1}{H} \sum_{I=1}^{n} h^{I} K_{i j}^{I},
$$

where $H$ is the total thickness of the preform and $h^{I}$ is the thickness of a ply $I(I=1,2, \ldots, n)$. 


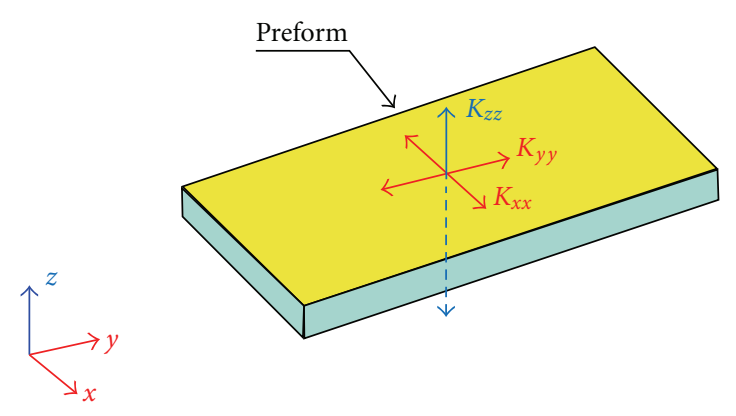

$\leftrightarrow$ Longitudinal permeability

$\longleftrightarrow$ Transverse permeability

Figure 5: Longitudinal and transverse permeabilities in a 3dimensional system.

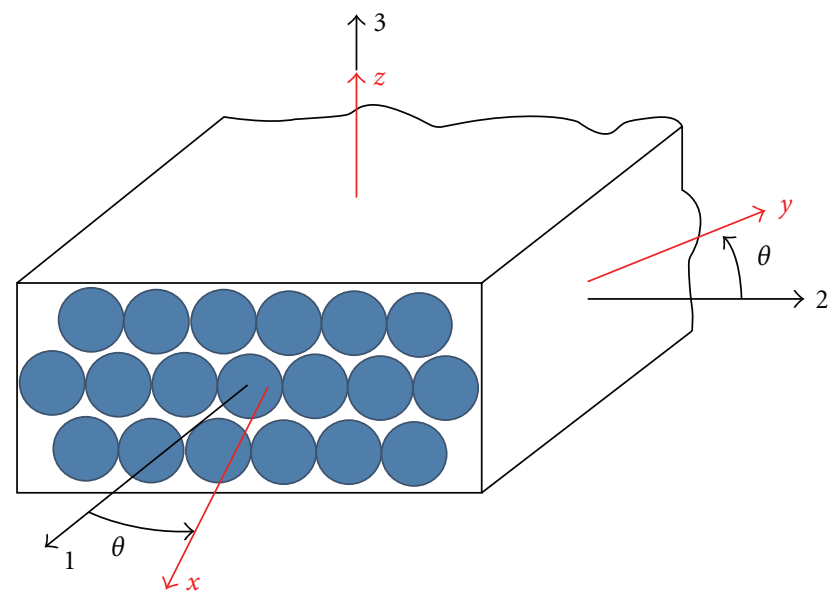

Figure 6: Principal and general coordinate systems of a fibrous porous medium.

Using a combination of Darcy's law and continuity equations yields the equation governing the pressure distribution, which can be written in compact form as

$$
\nabla \cdot\left(\frac{K}{\mu} \nabla P\right)=0
$$

Or in fully developed form, (12a) can be written as

$$
\begin{aligned}
& \frac{\partial}{\partial x}\left(\frac{K_{x x} \partial P}{\mu \partial x}\right)+\frac{\partial}{\partial x}\left(\frac{K_{x y} \partial P}{\mu \partial y}\right)+\frac{\partial}{\partial y}\left(\frac{K_{y x} \partial P}{\mu \partial x}\right) \\
& +\frac{\partial}{\partial y}\left(\frac{K_{y y} \partial P}{\mu \partial y}\right)=0 .
\end{aligned}
$$

The obtained system of equations can be solved by numerical approaches, where the corresponding boundary conditions of the system are generally defined as follows [18]:

(i) at the injection port: $P=P_{0}$ (constant pressure);

(ii) at the flow front: $P=P_{f}$ (atmospheric pressure, i.e., $1013.25 \mathrm{hPa}$ );

(iii) at the mould wall: $\partial P /\left.\partial n\right|_{\text {wall }}=0$.

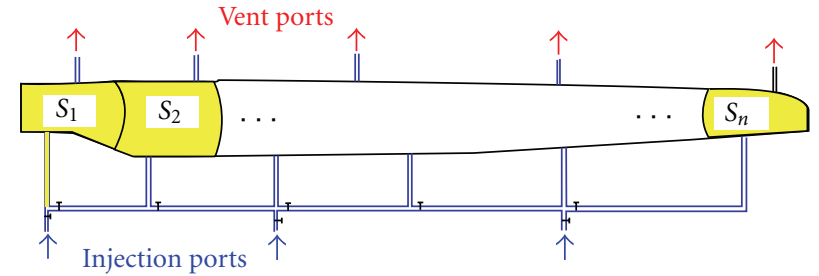

FIgure 7: Progressive impregnation of fibrous preforms by RTM sequential injection process.

A finite element method, employing a computer program, has been used in the current investigation.

It should be noted that this study is focused solely on a numerical analysis to give an idea about the behaviour of the resin flow in a flat anisotropic fibrous preform, characteristic of a wind turbine blade. It is clear that a particular inquiry should be paid to experimental study in order to conduct a test-calculation correlation. This issue will be investigated later and once the experimental results are available, their comment will be discussed in future publications.

\section{Results and Discussion}

For large-scale composite wind turbine blades, designed mainly for offshore applications, the resin injection is performed in a sequential manner [19]. In such RTM process simulation, the injection ports are located on the trailing edge, whereas the vent ports are located on the leading edge. Figure 7 shows the position of these ports and illustrates how the procedure is started. The injection process starts from the blade root, characterised by the section $S_{1}$. This latter corresponds to the most critical area, submitted during working conditions to high stress levels. Once the fibrous preform corresponding to section $S_{1}$ is saturated with resin, the injecting operation is then moved progressively to the next section $S_{2}$ and so on, until it reaches the last section $S_{n}$, corresponding to section relative to the free end of the wind turbine blade.

To further generalize the current moulding technique and get an idea about the resin flow behaviour during the resin filling stage, two unidirectional fibrous preforms are considered in this analysis. The first one was made up of onelayer thickness of $9 \mathrm{~mm}$ composed of a certain number of unidirectional plies, whereas the second one was made up of two layers with different fibre orientations and the thickness of each layer was assumed to be equal to $4.5 \mathrm{~mm}$. The fibrous preforms are in the form of square flat plates of dimensions $40 \mathrm{~cm} \times 40 \mathrm{~cm}$ and are considered to be extracted from the original blade structure (i.e., upper or lower surfaces corresponding to zone $\mathrm{B}$ ).

Figure 8(a) shows the positions of injection and vent ports; they are located at the midpoint of the mould edge and the opposite side, respectively.

The numerical simulation of resin flow behaviour through the fibrous preform during mould-filling stage is calculated using a finite element program based on (6a) and 


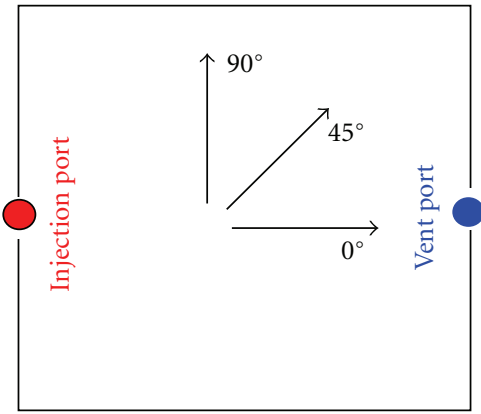

(a) Fibrous preform

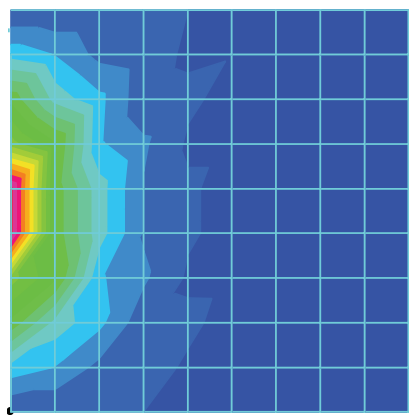

(c) Layer orientated at $\theta=90^{\circ}$

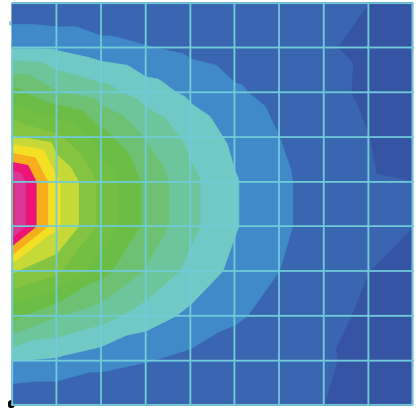

(b) Layer orientated at $\theta=0^{\circ}$

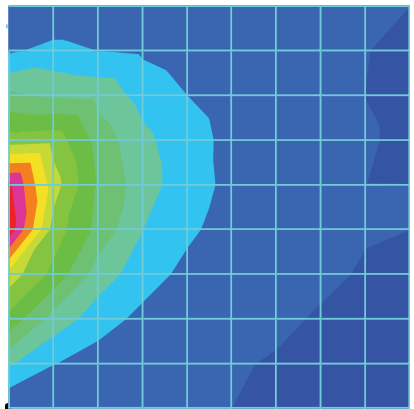

(d) Layer orientated at $\theta=45^{\circ}$

FIgURE 8: Numerical simulation of resin flow behaviour during mould filling stage for a single-layer fibrous preform.

(6b). The various concentrations of pressure field are given in the form of different colours, as shown in Figures 8(b)-8(d).

6.1. Simulation of Flow Resin Behaviour for the Case of One Unidirectional Layer. Three unidirectional fibre orientations (i.e., $\theta=0^{\circ}, \theta=90^{\circ}$, and $\theta=45^{\circ}$ ) were considered in this analysis and the output results are illustrated in Figure 8. It can be seen that the flow of the resin along the fibre direction $\theta=0^{\circ}$ (see Figure $8(\mathrm{~b})$ ) is more important than the other directions (Figures $8(\mathrm{c})$ and $8(\mathrm{~d})$ ). The reason for this is due to the presence of important volume fraction of pores in the longitudinal direction of fibres. These pores will provide the preferential paths of resin flow through the porous medium and will accelerate the process of impregnation. On the other hand, the flow rate is slow when fibres are orientated at $\theta=90^{\circ}$ (Figure $8(\mathrm{c})$ ). This can be explained by the fact that fibres are perpendicular to the direction of resin movement, and this will create a sort of barrier which prevents the resin from spreading easily because of a low presence of pores in such direction. Therefore, the idea of a concentric flow of resin with regard to the position of single-point injection is not truly representative for orthotropic unidirectional fibre reinforcements.

6.2. Simulation of Flow Resin Behaviour for the Case of Two Unidirectional Layers. For this case study, positions of injection and vent ports remain the same as the previous analysis and are illustrated in Figure 9(a). However, the number of layers forming the laminated fibrous preform is doubled and each layer is oriented at a specific angle. To this end, three case studies with different orientations of layers are considered in this analysis and are symbolised by the following staking sequences: $\left[45^{\circ} / 90^{\circ}\right],\left[45^{\circ} / 0^{\circ}\right],\left[90^{\circ} / 0^{\circ}\right]$, and $\left[45^{\circ} /-45^{\circ}\right]$. The output results of this investigation are presented in Figures 9(b)-9(d), from which it can be discerned that the resultant flow is dominant in the case where stacking arrangement presents a significant distribution of pores through the fibrous preform, which provides a better flow of resin (see Figure 9(c)). On the contrary, the drainage of resin is anisotropic, slower, and less important for the case shown in Figure 9(b). Further to the fibre direction, it should be pointed out that the permeability depends also on the stacking order of layers (mainly for the transverse permeability).

The choice of an appropriate stacking sequence is usually determined by a finite element structural analysis and the output numerical results must meet the criteria required by the new certification specifications put into effect. However, any change in the fibre orientation of layers may, in one hand, promote the process of drainage of the resin, but on the other hand, it may affect the mechanical properties of the material and therefore the stiffness and strength of the resulting blade product. The final choice of stacking sequence must meet simultaneously and favourably the conditions identified by the numerical calculations and those imposed by the principles governing the RTM process. This particular issue should be carefully studied before the implementation of the process.

It should be noted that the integration of exact values of permeability into simulation software for RTM process can yield an acceptable and reliable approach regarding the resin flow behaviour. In addition, curves representing the pressure 


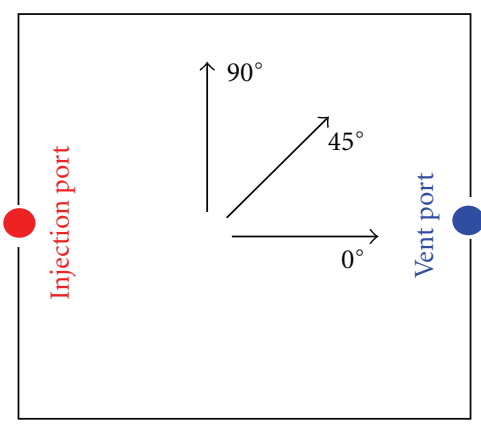

(a) Fibrous preform

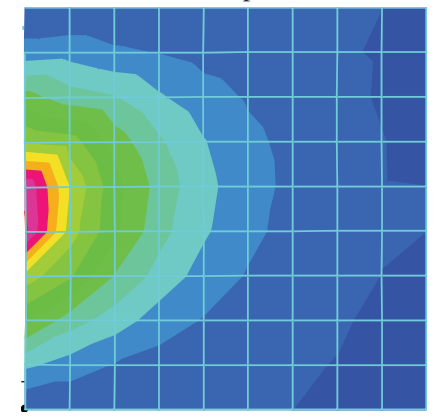

(c) Lay-up: $\left[45^{\circ} / 0^{\circ}\right]$

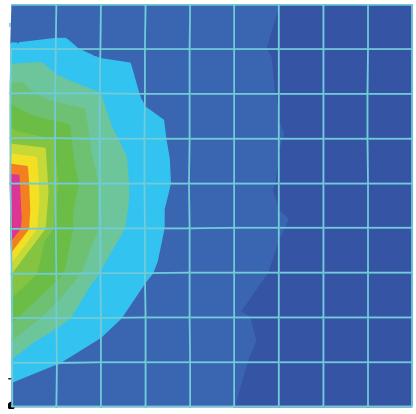

(b) Lay-up: $\left[45^{\circ} / 90^{\circ}\right]$

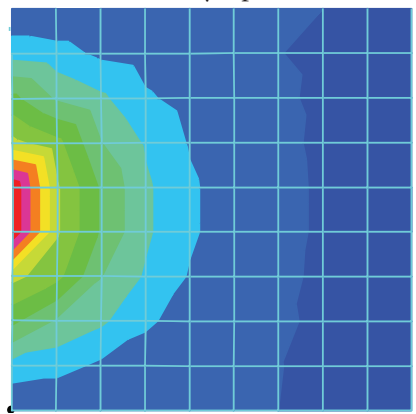

(d) Lay-up: $\left[90^{\circ} / 0^{\circ}\right]$

FIGURE 9: Numerical simulation of resin flow behaviour during mould filling stage for a double-layer fibrous preform.

field can be viewed for each ply constituting the preform during mould filling stage.

Future simulation software for RTM process should consider the ecological impacts discussed earlier in Section 1 and search for ways to promote green design; this is however not the case for some software and simulation codes.

Further to that, the shape of the blade structure, resin viscosity, temperature change, and location of injectionvent ports are other parameters that can influence the phenomenon of resin transfer and its flow behaviour. Other research and development studies are still needed to be performed on these parameters to better simulate the problem and get closer to the real case with a good testanalysis correlation.

\section{Conclusion}

Nowadays, quality assurance, health protection, and environmental preservation have become interdependent and interrelated aspects and are considered to be the primary factors that respond to the concept of sustainable development. In this context, the strategy to develop new generation of largescale offshore wind turbines is an important issue providing a key role in fighting climate change. However, the methods used to manufacture the turbine components, particularly the blades, must be green and environmentally friendly.

The key benefit that can be made from this analysis is that RTM process has an industrial solution for the production of composite wind turbine blades with cost-effective consideration and improved production rate. In addition, it is a clean process, less polluting, and allows considerable time saving compared to traditional manufacturing techniques.
Furthermore, the blade product is obtained in one shot with a total absence of bonding process of halfshells and has an excellent quality appearance on both sides and good mechanical properties. These advantages will undoubtedly attract the attention of relevant industrials and researchers and will boost innovation and creativity for the design of ultralarge wind turbine blades especially for future offshore applications.

Taking into account this ecological approach, the environmentally conscious designers, suppliers, and users will considerably encourage the progress and development of these manufacturing techniques, providing the industrial community with new methods and processes to be applied in modern technology of wind turbine blades.

The numerical analysis given in this paper reinforces the fact that numerical simulation of resin flow behaviour of complicated systems must not be dependent upon one analysis procedure only. Comprehensive modelling of a finite element solution from an experimental procedure should be undertaken before parameter studies are made.

\section{References}

[1] TPWind Secretariat, "2010-2012 Implementation Plan," Wind European Industrial Initiative Team, May 2010, http:// setis.ec.europa.eu/activities/implementation-plans/Wind_EII_ Implementation_Plan_final.pdf/view .

[2] B. Attaf and L. Hollaway, "Vibrational analyses of glassreinforced polyester composite plates reinforced by a minimum mass central stiffener," Composites, vol. 21, no. 5, pp. 425-430, 1990.

[3] B. Attaf, "Generation of new eco-friendly composite materials via the integration of ecodesign coefficients," in Advances in 
Composite Materials-Ecodesign and Analysis, B. Attaf, Ed., pp. 1-20, Intech, Rijeka, Croatia, 2011.

[4] B. Attaf, "Structural ecodesign of onshore and offshore composite wind turbine blades," in Proceedings of the lère Conférence Franco-Syrienne sur les Energies Renouvelables, Damascus, Syria, 2010.

[5] B. Attaf, "Eco-conception et développement des pales d'éoliennes en matériaux composites," in Proceedings of the 1er Séminaire Méditerranéen sur l'Energie Eolienne, Algeria, 2010.

[6] D. Cairns, J. Skramstad, and T. Ashwill, "Resin transfer molding and wind turbine blade construction," Technical Note SAND99-3047, Sandia National Laboratories, California, Calif, USA, 2000.

[7] S. G. Advani, Flow and Rheology in Polymer Composites Manufacturing, Composite Materials Series, Elsevier Science, Amsterdam, The Netherlands, 1994.

[8] B. Attaf, "Towards the optimisation of the ecodesign function for composites," JEC Composites Magazine, vol. 34, no. 42, pp. 58-60, 2007.

[9] B. Attaf, "Probability approach in ecodesign of fibrereinforced composite structures," in Proceedings of the Congrès Algérien de Mécanique (CAM '09), Biskra, Algeria, 2009.

[10] S. M. Habali and I. A. Saleh, "Technical note: design and testing of small mixed airfoil wind turbine blades," Renewable Energy, vol. 6, no. 2, pp. 161-169, 1995.

[11] K. M. Pillai, "Governing equations for unsaturated flow through woven fiber mats. Part 1. Isothermal flows," Composites A, vol. 33, no. 7, pp. 1007-1019, 2002.

[12] C. Nardari, B. Ferret, and D. Gay, "Simultaneous engineering in design and manufacture using the RTM process," Composites A, vol. 33, no. 2, pp. 191-196, 2002.

[13] H. Darcy and V. Dalmont, Les Fontaines Publiques de la Ville de Dijon, Paris, France, 1856.

[14] Y. Luo, I. Verpoest, K. Hoes, M. Vanheule, H. Sol, and A. Cardon, "Permeability measurement of textile reinforcements with several test fluids," Composites A, vol. 32, no. 10, pp. 1497-1504, 2001.

[15] A. Shojaei, S. R. Ghaffarian, and S. M. H. Karimian, "Threedimensional process cycle simulation of composite parts manufactured by resin transfer molding," Composite Structures, vol. 65, no. 3-4, pp. 381-390, 2004.

[16] K. Hoes, D. Dinescu, H. Sol et al., "New set-up for measurement of permeability properties of fibrous reinforcements for RTM," Composites A, vol. 33, no. 7, pp. 959-969, 2002.

[17] C. H. Park, W. I. Lee, W. S. Han, and A. Vautrin, "Weight minimization of composite laminated plates with multiple constraints," Composites Science and Technology, vol. 63, no. 7, pp. 1015-1026, 2003.

[18] H. Jinlian, L. Yi, and S. Xueming, "Study on void formation in multi-layer woven fabrics," Composites A, vol. 35, no. 5, pp. 595-603, 2004.

[19] P. Desfilhes, "Composites-l'utomatisation des grandes pieces," L'Usine Nouvelle, no. 2819, pp. 48-50, 2002. 

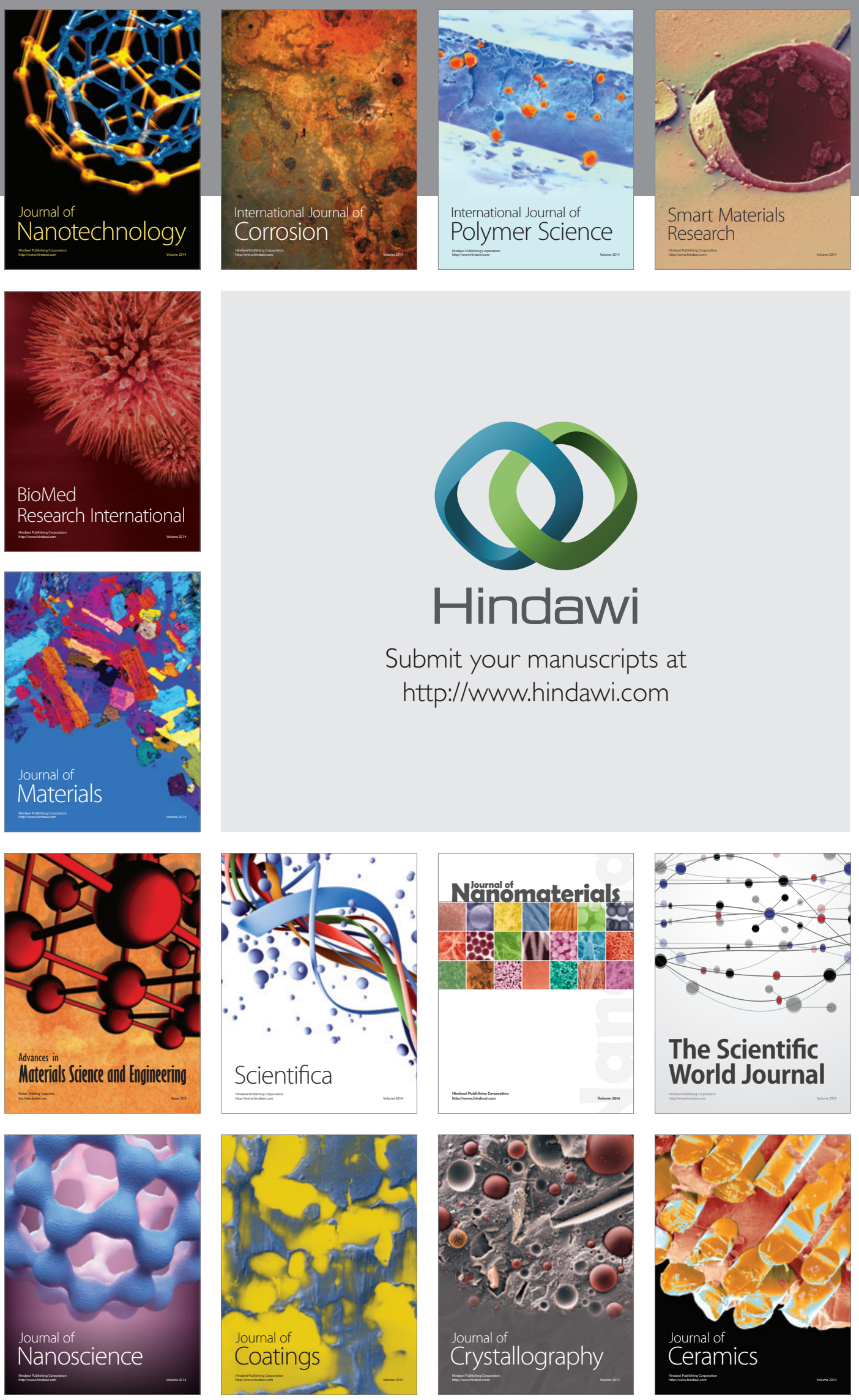

The Scientific World Journal

Submit your manuscripts at

http://www.hindawi.com

\section{World Journal}

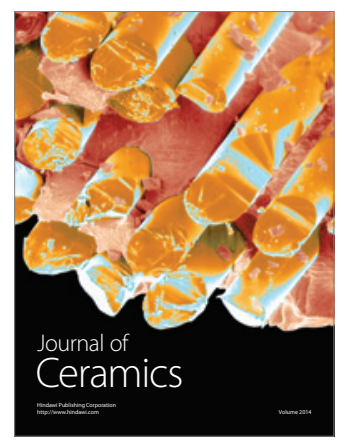

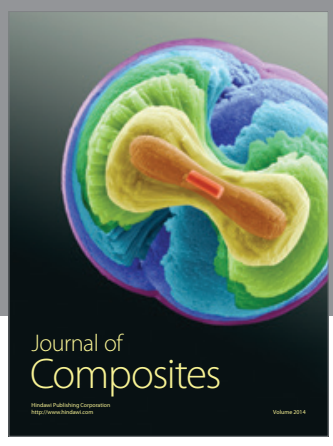
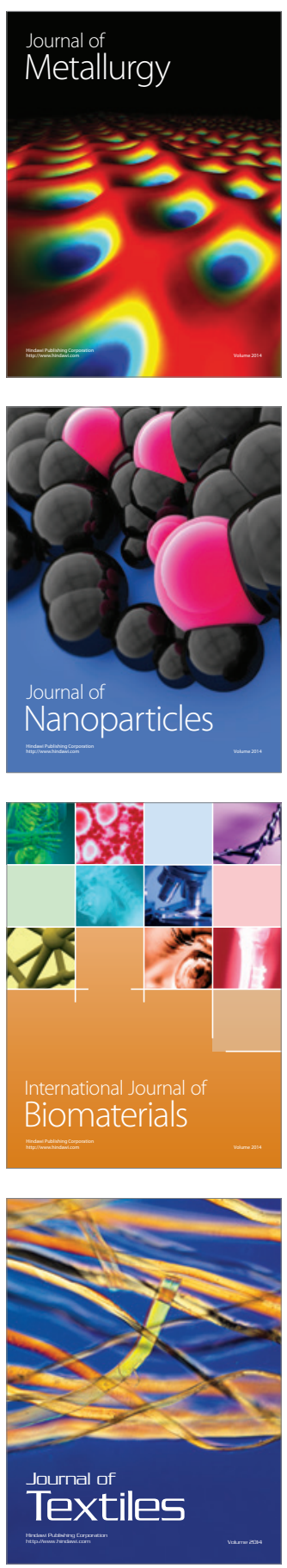